\title{
The treatment of cavernous sinus meningiomas: evolution of a modern approach
}

\author{
Daniel R. Klinger, M.D., Bruno C. Flores, M.D., Jeremy J. Lewis, M.D., \\ and SAmuel L. Barnett, M.D. \\ Department of Neurological Surgery, University of Texas Southwestern Medical Center, Dallas, Texas
}

\begin{abstract}
Cavernous sinus meningiomas (CSMs) are challenging lesions for the skull base neurosurgeon to manage given their close association with cranial nerves II-VI and the internal carotid artery. In the 1980s and early 1990s, with advancements in microsurgical techniques, increasing knowledge of the relevant microsurgical neuroanatomy, and the advent of advanced skull base surgical approaches, the treatment of CSMs involved attempts at gross-total resection (GTR). Initial fervor for a surgical cure waned, however, as skull base neurosurgeons demonstrated the limits of complete resection in this region, the ongoing issue of potential tumor recurrences, and the unacceptably high cranial nerve and vascular morbidity associated with this strategy. The advent of radiosurgery and its documented success for tumor growth control and limited morbidity in cavernous lesions has helped to shift the treatment goals for CSMs from GTR to tumor control and symptom relief while minimizing treatment- and lesion-associated morbidity. The authors review the relevant microanatomy of the cavernous sinus with anatomical and radiographic correlates, as well as the various treatment options. A modernized, multimodality treatment algorithm to guide management of these lesions is proposed.
\end{abstract}

(http://thejns.org/doi/abs/10.3171/2013.9.FOCUS13345) \begin{tabular}{l}
$\begin{array}{l}\text { KEY WORDS } \\
\text { skull base }\end{array} \quad \bullet \quad$ cavernous sinus meningioma $\quad \bullet \quad$ radiosurgery $\quad \bullet \quad$ microsurgery \\
\hline
\end{tabular}

$\mathrm{M}$ ENINGIOMAS involving the cavernous sinus are among the most challenging lesions for the skull base neurosurgeon to manage, given their potential involvement with cranial nerves II-VI and the internal carotid artery (ICA). The treatment of cavernous sinus meningiomas (CSMs) has evolved over the past 30 years, as there has been a growing understanding of the relevant skull base anatomy, the neurovascular risks of aggressive approaches, and the adoption of safe alternative or adjunctive treatments in the form of radiotherapy and radiosurgery. Modern treatment strategies attempt to preserve neurological function and limit tumor proliferation by using surgery and radiation therapy in a safe and effective manner.

Modern anatomical study of the cavernous sinus dates to the pioneering work of Parkinson and Ramsay in the 1960s. ${ }^{37-39}$ These cadaveric studies and improvement in microsurgical technology and surgical techniques allowed Dolenc, Hakuba, and Kawase to propose novel skull base approaches to the cavernous sinus..$^{10,13,20,43} \mathrm{En}$ -

Abbreviations used in this paper: $\mathrm{CSM}=$ cavernous sinus meningioma; GKRS = Gamma Knife radiosurgery; GTR = gross-total resection; ICA = internal carotid artery; SRS = stereotactic radiosurgery; STR = subtotal resection. thusiasm for skull base approaches seeking complete removal of CSMs peaked in the late 1980s and early 1990s as neurosurgeons gained considerable experience with surgical treatment of these lesions. Despite these advances, concerns remained regarding cranial nerve morbidity, ischemic complications from vascular sacrifice and bypass, and the unsettling knowledge that long-term followup of these patients continued to demonstrate cases of recurrence. $6,21,33,47$

With its proliferation in the early 1990s, stereotactic radiosurgery (SRS) became an attractive treatment alternative or adjunct for the skull base neurosurgeon..$^{12,16,24,50} \mathrm{As}$ knowledge of the inherent morbidity of attempted radical resection of CSMs became more accepted, treatment strategies involving either primary radiosurgery of CSMs or subtotal resection (STR) followed by adjunctive Gamma Knife radiosurgery (GKRS) have become more prevalent. Currently, our detailed understanding of the microsurgical anatomy of the cavernous sinus along with modern MRI of the cavernous sinus and CSMs can facilitate a multimodality treatment strategy that minimizes treatment morbidity while attempting to maximize tumor control. These treatment options generally include observation, primary radiosurgery, or STR, with either continued observation or follow-up radiosurgery of residual disease. 


\section{Anatomy}

The cavernous sinuses are paired venous structures seated at the middle cranial base surrounding the midline sella turcica. ${ }^{54}$ The bony floor of the sinuses is formed by the sphenoid and temporal bones. Rostrally, the sinus extends from the inferior surface of the anterior clinoid process anteriorly to the posterior clinoid process posteriorly. At the skull base caudally, the cavernous sinus extends from the inferior edge of the superior orbital fissure to the superior end of the petroclival fissure. The medial border of the cavernous sinus is the bony floor and lateral wall of the sella. Laterally, it abuts the medial temporal lobe. As the dura of the floor of the middle cranial fossa curves rostrally and medially to form the lateral wall of the sella, the layers diverge to form a sleeve that houses the critical structures of the cavernous sinus. The cavernous sinus proper contains the cavernous segment of the ICA and its branches, the sympathetic plexus and cranial nerves III, IV, and VI, and the first and second divisions of cranial nerve V (V1 and V2). Cranial nerves III and $\mathrm{IV}$, and the $\mathrm{V} 1$ and $\mathrm{V} 2$ divisions travel in the lateral wall of the cavernous sinus, with cranial nerve III occupying the most rostral position and V2 the most caudal position. Cranial nerve VI occupies a more medial position in the cavernous sinus proper. As a venous channel, the cavernous sinus also receives multiple venous tributaries, including the superior and inferior petrosal sinuses, the basilar sinus, the intercavernous sinus, as well as various draining veins of the skull base, sylvian fissure, and middle cerebral vein.

An understanding of the dural anatomy of the cavernous sinus can help facilitate the use of a successful treatment strategy for CSMs that minimizes treatment morbidity. The dura of the middle fossa floor consists of an inner layer adherent to the bone, an "endosteal" or periosteal layer, and an outer layer that faces the temporal lobe and meninges (a meningeal layer). ${ }^{54}$ el-Kalliny et al. described these endosteal and meningeal dural layers as the inner membranous and outer dura propria layers, respectively. ${ }^{8}$ As these dural layers of the middle fossa floor curve rostrally to form the lateral wall of the cavernous sinus, they separate into surgically distinct planes. The endosteal layer forms the inner lateral wall of the cavernous sinus and contains within it cranial nerves III, IV, and V1 and V2. Lateral to this distinct layer is the meningeal layer that abuts the intradural intracranial space, containing the meninges and medial temporal lobe. The lateral sinus wall, or meningeal layer, blends into the dura lining Meckel's cave. The inner endosteal layer covers the contents of the superior orbital fissure and is continuous with the periorbita. It is possible both in anatomical and surgical dissection to safely develop the plane between the endosteal and meningeal dural layers of the lateral wall of the cavernous sinus. . $^{1,8,10,11}$ This dissection is critical in CSM surgery because it may allow safe resection of all, or a portion of, tumor that has essentially grown in the "intradural" space, between the leaflets of the endosteal and meningeal dural layers. In general, microsurgical strategies that minimize perioperative morbidity for CSMs will respect the inner endosteal dural layer, thus minimizing any risk to the critical neurovascular structures of the sinus itself. Careful review of MRI of CSMs can delineate tumor involvement of the sinus itself and, therefore, influence the mode of treatment. Figure $1 \mathrm{dem}-$ onstrates the MRI findings of CSMs that demonstrate both a large intracavernous component and those that are confined to a predominantly extracavernous component.

Meningiomas of the cavernous sinus are an anatomically heterogeneous group of skull base tumors. ${ }^{1,8,46}$ They classically arise from the dura of the cavernous sinus itself. However, many modern series of CSMs include meningiomas that likely arise from the dura of the sphenoid ridge, clinoid processes, petroclival region, or anterior skull base, and extend into, or infiltrate, the cavernous sinus. ${ }^{1,46}$ Cavernous sinus meningiomas also may vary significantly in their degree of involvement, or invasion, of the sinus. Cavernous sinus meningiomas may grow within the plane of the "intradural" space, between the layers of cavernous sinus dura, without invading the cavernous sinus proper. They may infiltrate the sinus itself, surrounding the cranial nerves and cavernous ICA, or invade the venous sinus. Histological studies of invasive CSMs that were resected within the cavernous sinus proper have demonstrated that these tumors may invade cranial nerves and the ICA wall itself..$^{25,26,43}$ These findings call into question the utility of microsurgically exploring the cavernous sinus proper if tumor control may be achieved with less invasive and less morbid means.

\section{Natural History and Pathology}

The natural history of CSMs is difficult to ascertain. It is believed that cavernous sinus tumors represent $1 \%$ of all intracranial tumors, $41 \%$ of which are meningiomas. ${ }^{42}$ A Canadian population-based study from 1980 to 1985 found that $22 \%$ of primary intracranial tumors were meningiomas. ${ }^{2}$ The prevalence of meningiomas in the general population is believed to be approximately $1-5$ per 100,000 in men, and 2-7 per 100,000 in women., ${ }^{2,53}$ Female sex and advanced age are associated with a higher incidence of meningiomas, and many large population studies have noted a higher incidence when autopsy specimens are included. ${ }^{19}$ While many neurosurgical series may be biased toward clinically symptomatic lesions and data on conservative follow-up of meningiomas are
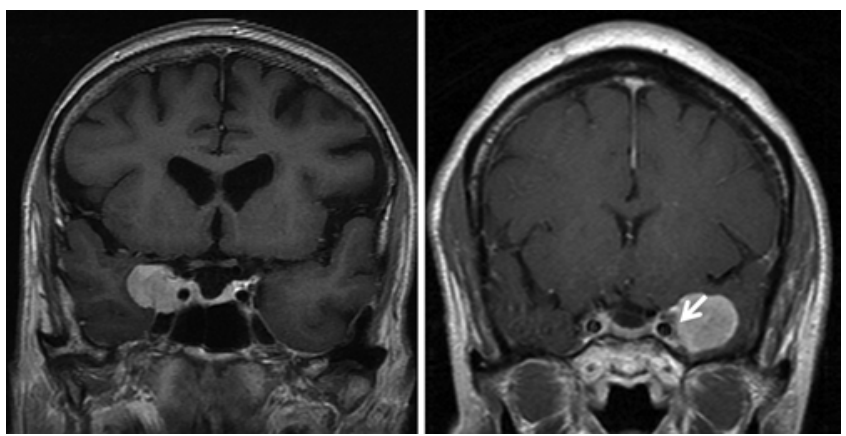

FIG. 1. Gadolinium-enhanced coronal MR images showing CSMs with intracavernous (left) and predominantly extracavernous (right) components. The arrow delineates the lateral dura distinguishing the cavernous sinus proper from the extracavernous tumor. 


\section{Evolution in treatment of cavernous sinus meningiomas}

scarce, a number of recent small studies of asymptomatic meningiomas demonstrates growth over an average of several years' follow-up. Most growth rates are estimated to be on the order of 0.02 to $0.24 \mathrm{~cm} /$ year. ${ }^{31,34} \mathrm{~A}$ recent natural history study of asymptomatic meningiomas followed conservatively with repeat imaging noted $44 \%$ of 277 tumors demonstrated linear tumor growth at a mean follow-up of 3.8 years. ${ }^{35}$ When including tumors subjected to volumetric analysis, the percentage of meningiomas that grew in this series was $77 \%$. Older patients with small $(<25 \mathrm{~mm})$ calcified meningiomas were less likely to demonstrate progression. There may be some suggestion that asymptomatic skull base meningiomas grow at a slower rate than meningiomas at other locations. Hashimoto et al. conducted MRI volumetric analysis of 113 incidentally discovered meningiomas, 38 of which originated at the skull base. ${ }^{15}$ They found that after approximately 4 years of follow-up, nearly $75 \%$ of non-skull base meningiomas demonstrated at least $15 \%$ growth, whereas only $34 \%$ of skull base meningiomas did. Considerably longer-term follow-up of a surgical series with STR also demonstrated a not insubstantial rate of recurrence of $15 \%-100 \% .22,28,49$ Most tellingly, the recurrence rates tend to increase with increasing follow-up ( $>15$ years) and with larger residual tumor remnant (higher Simpson grade). ${ }^{18}$

Meningiomas originating from the cavernous sinus tend to cause symptoms by compressing or invading nearby neurovascular structures. Given their close proximity to cranial nerves II-VI and the ICA, CSMs often present with neurological deficit. Patients with CSMs most frequently present with ocular motor deficits such as diplopia, anisocoria, ophthalmoplegia, and ptosis. Optic pathway compression can cause visual field deficits. Optic canal invasion or optic nerve compression secondary to hyperostosis can lead to ipsilateral loss of visual acuity. Facial numbness, pain, and dysesthesias can result from compression of 1 or multiple branches of cranial nerve V. Pituitary stalk and gland dysfunction, though uncommon, can result from tumor compression and may require endocrine laboratory workup. Patients with tumors arising from the sphenoid wing that infiltrate the cavernous sinus may present with seizures or hemiparesis. Finally, headaches are commonly associated with CSMs.

\section{Surgical Treatment}

There has been an evolution in thought and approach in the surgical strategies of CSM removal. Aggressive resection of CSMs, with the aim of entering the sinus to ensure gross-tumor removal, which was once proposed to be the definitive treatment standard, has fallen out of favor for a number of reasons. Initial enthusiasm with aggressive resection of CSMs has been tempered by reports of significant procedure-related neurological morbidity, the persistent difficulty of achieving a gross-total resection (GTR), and the continued need for follow-up and concern for persistent risk of recurrence. In a review of 39 surgically treated CSMs, O'Sullivan et al. reported on 8 patients who underwent complete tumor removal. ${ }^{33}$ Their rate of new cranial nerve deficit was $17.6 \%$, and only 2 patients experienced improved cranial nerve func- tion postoperatively. Additionally, in 7 of the 8 patients, there was preoperative tumor encasement of the cavernous ICA (Hirsch Grade 2 or higher), which often required a preoperative balloon occlusion test with resulting vessel sacrifice with or without bypass. DeMonte et al. reported on 41 patients who underwent aggressive resection of CSMs. ${ }^{6}$ Gross-total resection was achieved in $76 \%$, but $5 \%$ developed recurrence at 5-year follow-up from GTR. Seven patients experienced 10 new cranial nerve deficits; 2 patients died postoperatively, 1 from severe delayed vasospasm and 1 secondary to complications from hypothalamic infarction. De Jesús et al. described a GTR in $61 \%$ of 119 CSMs. ${ }^{5}$ Two patients in their series suffered cerebral infarcts from ICA injury, and the median postoperative Karnofsky Performance Scale score dropped from 90 to 80 .

As surgical experience with aggressive resection of CSMs lost favor given the untenable rates of morbidity, and as radiosurgery gained favor as a primary and adjunct treatment modality, more recent series focused on rates of tumor control with STR.,40,51 Abdel-Aziz et al. presented a series of 38 large sphenoid-wing meningiomas involving the cavernous sinus. ${ }^{1}$ These investigators achieved complete microscopic excision in 22 of 24 patients with tumors only involving the lateral compartment of the sinus (Hirsch Grades 0 and 1). Subtotal resection was performed in 14 patients with holocavernous tumor infiltration surrounding the cavernous ICA (Hirsch Grade 2-4). The morbidity rate was $16 \%$ in this study, with ocular nerve dysfunction improved in $40 \%$ of patients postoperatively. They found a $6 \%$ recurrence rate for completely excised tumors and an $8 \%$ regrowth rate in the STR subgroup. Sindou et al. described a series of 100 patients with CSMs, 88 of whom underwent either extracavernous tumor resection alone or extracavernous resection with a partial intracavernous debulking. ${ }^{47}$ They found disorders of vision, ocular motility, and trigeminal function were statistically more likely to be disturbed postoperatively when surgery extended into the cavernous sinus proper. They demonstrated that the tumor remnant did not regrow in $87 \%$ of the 88 patients who underwent STR, and regrowth was noted in only $13 \%$ at a mean follow-up of approximately 8 years.

\section{Radiotherapy}

Concurrent with a developing understanding of the limits and risks of microsurgical resection of CSMs, radiosurgery has evolved as an excellent primary and adjunct treatment modality in these lesions. ${ }^{16,23,24,32,44,50,51}$ Stereotactic radiosurgery has demonstrated very good rates of tumor control as well as low rates of postprocedural morbidity, particularly cranial nerve injury. The majority of studies of SRS for CSMs are retrospective single-institution case series. ${ }^{7,23,32}$ In the majority of radiosurgical series involving either primary SRS or adjunctive SRS after microsurgical resection, the tumor volume is less than $15 \mathrm{~cm}^{3}$ and often less than $10 \mathrm{~cm}^{3}$. Progression-free survival rates at 5 and 10 years were $87 \%-99 \%$ and $73 \%-94 \%$, respectively. Stereotactic radiosurgery also includes radiographic tumor control, documented in a 
clear subset of patients. ${ }^{32,41}$ Most studies demonstrate a 29\%-69\% radiographic response rate, with tumor shrinkage in as many as $40 \%$ of treated CSMs. Largely consistent with these biological responses are documented rates of clinical response or improvement after SRS. Improvement in cranial nerve deficits are typically in the range of $20 \%-42 \%$ after either primary or adjunctive SRS.

Accepted radiosurgical dosing rates to surrounding critical neurovascular structures is what largely determines the effective SRS tumor dose margin for CSMs, which likely lies somewhere between 12 and $16 \mathrm{~Gy}{ }^{41}$ Tishler et al. reported no complications in patients treated with a maximal dose to the cavernous sinus of less than $25 \mathrm{~Gy}$ and found no visual complications in patients who received less than $10 \mathrm{~Gy}$ to the optic apparatus. ${ }^{52}$ More modern treatment planning and adopting the strategy of limiting the SRS dosage to small segments of the optic pathway have allowed Morita and colleagues to treat tumor margins abutting small segments of the optic nerve with doses up to 16 Gy without negative sequelae; however, they did note 6 new cases of trigeminal neuropathy with a treatment dose of 19 Gy to the region of Meckel's cave in their study. ${ }^{30}$

In general, however, it appears that for small CSMs, the rates of morbidity are significantly less with SRS than those noted with direct microsurgical resection, although it is likely that the true benefit is at least partially obscured by a selection bias at work, in which large symptomatic lesions are often treated using surgery and smaller, minimally symptomatic lesions prove to be ideal radiosurgical cases. A recent meta-analysis of more than 2000 CSMs retrospectively evaluated and treated with either GTR, STR followed by adjuvant postsurgical radiotherapy/radiosurgery, or SRS alone demonstrated a significantly higher rate of cranial nerve morbidity in patients undergoing resection as compared with those undergoing SRS (59.6\% vs $25.7 \%$, respectively). ${ }^{50}$ Most recent large SRS series have demonstrated durable radiosurgical complication rates of less than 15\%; the majority of lasting deficits reported in the literature include cranial nerve deficit, motor deficit, hydrocephalus, vascular injury and subsequent ischemic event, hypothalamic dysfunction, perilesional edema, and memory deficits.

Although receiving less consideration in recent years with the growth of SRS, fractionated radiotherapy remains an additional viable treatment option for CSMs. A recent review of retrospective series of CSMs treated with fractionated radiotherapy found rates of progressionfree survival of $89 \%-94 \%$ at 5 years and $76 \%-94 \%$ at 10 years posttreatment. Typical safe and effective treatment protocols involve average total dosing of 50-55 Gy, involving 30 fractions of 1.6-1.9 Gy per treatment. ${ }^{45}$ Some centers may reserve fractionated therapy for larger tumor volumes less likely to be amenable to single-treatment SRS, with safe tumor margin dosing in the range of 12-16 Gy. One recent study in 2005 from Metellus et al. compared 2 patient subgroups with CSMs treated with either primary or adjuvant SRS or fractionated radiotherapy. ${ }^{29}$ They achieved comparable rates of tumor growth control in both groups (>90\%). They noted a significantly increased radiobiological response in the SRS group of
$53 \%$ tumor shrinkage in comparison with $29 \%$ tumor shrinkage in the fractionated radiotherapy group, which is largely consistent with results from other series. They argued that this more favorable radiological response, as well as the convenience for patients and practitioners of a single treatment session with SRS, argues for SRS as a primary radiotherapeutic option in amenable cavernous sinus lesions.

\section{Modern Multimodality Therapy}

With the progression of our understanding of the treatment complexities of CSMs, the goals of treatment have become more clearly delineated. An oncological cure with radical resection is usually not possible; numerous series delineate that the risks of tumor recurrence persist even after GTR. Additionally, the risk of increased cranial nerve and vascular morbidity after exploration of the cavernous sinus proper is too high. Therefore, the goal of treatment must entail control of tumor progression while maximizing neurological function for the patient, both by improving or stabilizing existing deficits and by minimizing treatment-related morbidity. Figure 2 provides a simple algorithm to determine which CSMs warrant treatment. As is always the case in meningioma treatment, treatment decisions must be informed by an understanding of the patient's biology and desires (including age, comorbidities, and functional status) as well as the tumor's natural history and pathology. Symptomatic CSMs and those with documented radiographic progression likely warrant treatment in most cases. In Fig. 3, we propose a modern treatment algorithm in which anatomical and radiographic characteristics of CSMs influence the treatment modality. Small $\left(<20-30 \mathrm{~cm}^{3}\right)$, primarily intracavernous lesions are ideal candidates for radiosurgery. Multiple studies have demonstrated greater than $90 \%$ tumor control at 5-10 years after treatment, with at least $30 \%-40 \%$ of patients experiencing improvements in tumor-related neurological deficits with, on average, a less than $12 \%$ treatment-related morbidity. ${ }^{14,27,41,44,48}$

Similarly, large meningiomas of the skull base that either originate from, or have some involvement with, the cavernous sinus likely require initial surgical treatment with tumor resection or debulking. Given their growth patterns, many of these lesions exhibit mass ef-

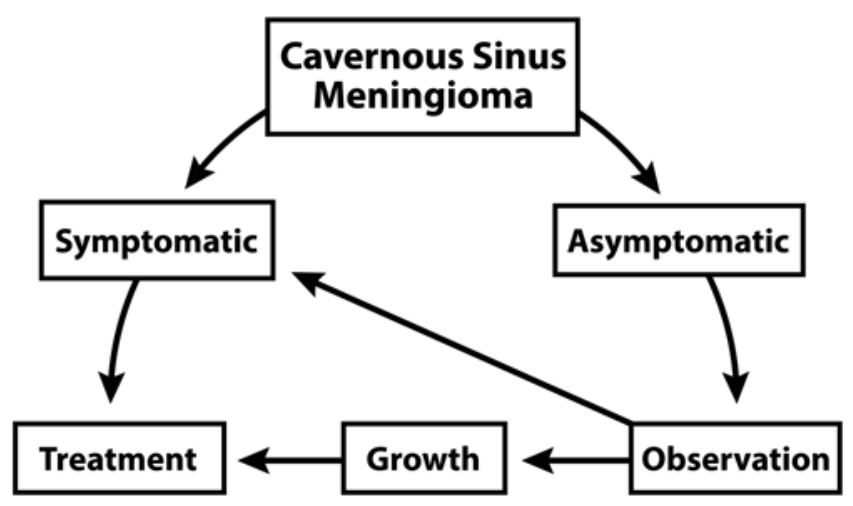

FIG. 2. Diagram of the algorithm used to determine treatment versus observation of CSMs. 


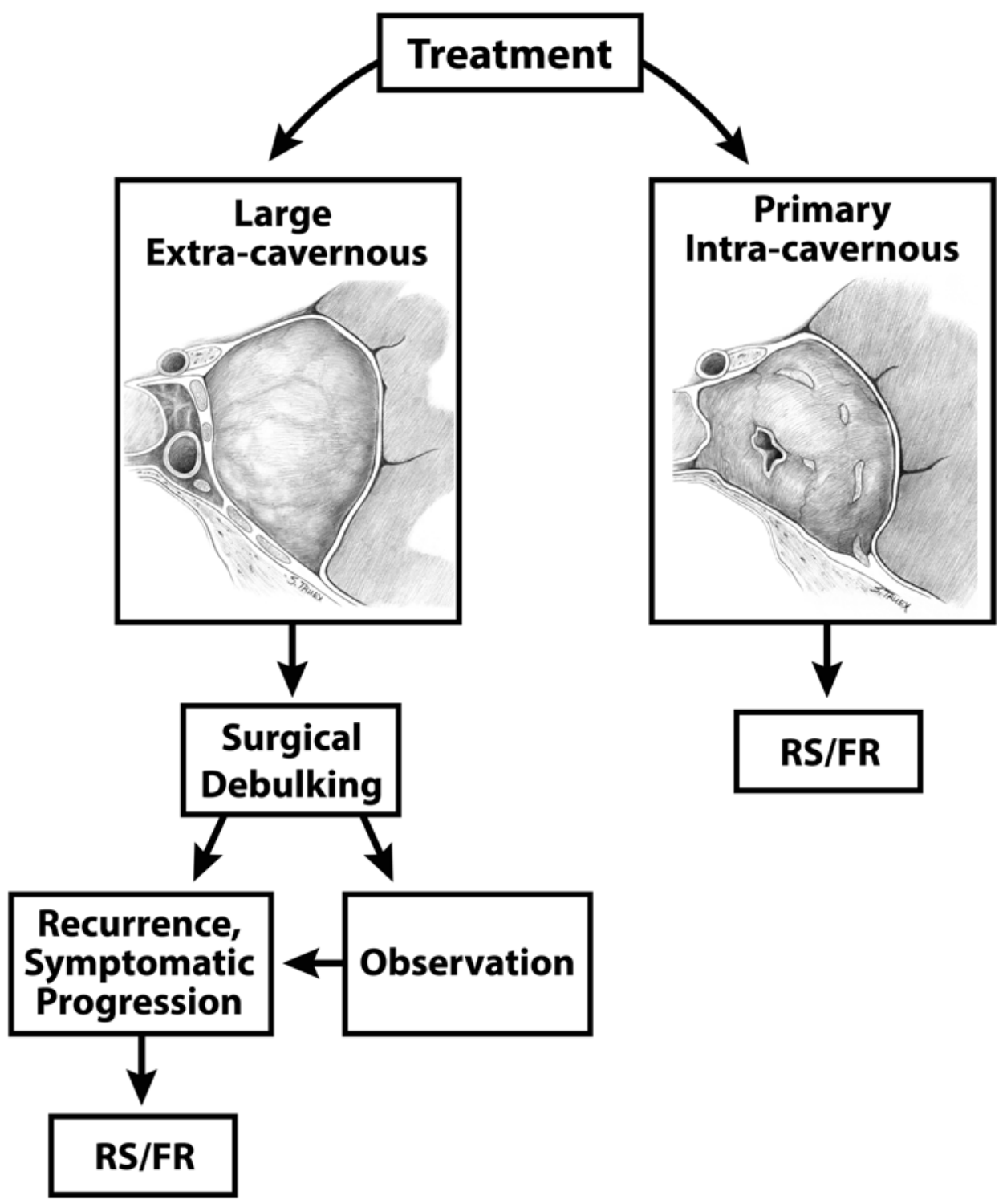

FIG. 3. Multimodality treatment algorithm for CSMs. The illustration on the left of the algorithm depicts a CSM with a large extracavernous component. The illustration on the right of the algorithm depicts a CSM with a large intracavernous component. FR = fractionated radiotherapy; RS = radiosurgery. Copyright Suzanne Truex. Published with permission.

fect on, or invasion of, the temporal lobe, diencephalon, or optic canal. The surgical approach should be tailored to the offending pathology. At minimum, this involves a frontotemporal craniotomy; sphenoid wing drilling; and exposure of the middle cranial fossa, superior orbital fissure, and lateral cavernous sinus. Variations may include orbital and zygomatic osteotomies, opening of the optic canal, or removal of the anterior clinoid process.

Recent surgical series have demonstrated that a transition from radical resection with exploration of the cavernous sinus proper, to a more conservative surgical strategy that involves removal of tumor from the "interdural" lateral aspect of the cavernous sinus without exenterating the sinus proper, yields improved results. ${ }^{27,36,40,46}$ In a modern series of large ( $>3 \mathrm{~cm}$ diameter) sphenocavernous meningiomas, Abdel-Aziz et al. detailed a surgical strategy that involved tumor debulking with removal of tumor within the interdural lateral compartment of the cavernous sinus, with tumor remnant treated with adjunctive linear accelerator radiosurgery or fractionated radiotherapy, and found a $90 \%$ tumor control rate in those tumors treated with adjuvant radiation. ${ }^{1}$ They also noted that the rate of recurrence was similar (at $6 \%$ in both patient subgroups) in subtotally resected tumors and those believed to be a microscopic GTR. Pamir et al. compared CSMs treated at their institution in the pre-GKRS era (prior to 1997) and after the introduction of GKRS. ${ }^{36} \mathrm{In}$ the pre-1997 patients, a surgical strategy of radical resection with cavernous sinus exploration was employed. After 1997, these investigators practiced surgical removal of the extracavernous sinus tumor component with GKRS treatment of the intracavernous remnant. At 3 years' follow-up, they found the modern conservative approach yielded better tumor control and less cranial nerve mor- 
bidity. Finally, an Italian series by Pichierri et al. compared a modern subgroup of CSMs that were treated by conservative resection (which they termed the "closed sinus" group) with a subgroup of CSMs that were radically resected (the "open sinus" group) and found no differences at a mean follow-up of 9.7 years with regard to tumor recurrence or progression. ${ }^{40}$ They did note a higher rate of early and permanent postoperative morbidity in the "open sinus" group. They demonstrated that those tumors that underwent STR with adjuvant radiosurgery had an $81.5 \%$ rate of clinicoradiological stability.

The major persistent dilemmas regarding the treatment of CSMs involve tumors of medium to large size $\left(>20 \mathrm{~cm}^{3}\right)$, particularly those with close proximity to the optic apparatus. The literature provides data on the efficacy of multiple treatment options for these tumors. ${ }^{1,40,41,48}$ One option for treatment of larger CSMs includes debulking the tumor surgically with a plan for adjuvant radiosurgery or radiotherapy. The surgical goal often involves reducing tumor volume to allow effective radiosurgical treatment with minimal radiation-induced side effects and decompression of the optic apparatus to provide a buffer for safe radiosurgery. Couldwell et al. reported their experience in 11 patients treated with conservative surgical debulking and then by follow-up radiosurgery or intensity-modulated radiation therapy. ${ }^{4}$ These authors attempted to achieve a minimum postoperative 5-mm interpositional distance between the residual tumor and optic apparatus. In their small series, they noted some degree of clinical improvement after treatment in $45 \%$ of their patients, with no permanent deficits and radiological tumor control in all 11 patients. A modern Turkish patient series proposed an algorithm for initial surgical versus radiosurgical treatment. ${ }^{36}$ In patients with tumors less than $20 \mathrm{~cm}^{3}$ and greater than $3 \mathrm{~mm}$ from the optic system, they proposed primary GKRS as treatment. In tumors greater than $20 \mathrm{~cm}^{3}$ and less than $3 \mathrm{~mm}$ from the optic system, they advocated surgical debulking of the extracavernous tumor for planned follow-up GKRS. They found greater clinical improvement, improved tumor control, and fewer treatment-related side effects with this strategy in comparison with their historical strategy of primarily resection for cure. Some have argued, however, that rates of neurological improvement are higher when radiosurgery is used as a primary treatment for CSMs than as an adjuvant treatment, presumably secondary to the iatrogenic insult that comes from surgical devascularization of the lateral sinus and its nerve structures. ${ }^{32}$

Other treatment strategies for larger CSMs include primary SRS (often at a lower dose or margin dose), multisession SRS, or fractionated radiotherapy.,17,48 With larger tumor treatment volume, there is a clear trade-off between maintaining higher margin dosing (of 14-16 Gy) with likely higher radiation-related complications, versus accepting a lower rate of tumor growth control. Pollock et al. noted a $3 \%$ rate of radiation-related complications in tumor volumes of no more than $9.3 \mathrm{~cm}^{3}$ compared with a radiation complication rate of $21 \%$ for treated tumor volumes greater than $9.3 \mathrm{~cm}^{3}$, the most frequent of these complications being trigeminal nerve dysfunction. ${ }^{41}$ Other studies have validated this association of increased radiation-related com- plications with increased treated tumor volumes. ${ }^{55}$ Some centers have addressed this association by reducing total tumor or tumor-margin treatment volume, staging SRS treatment, or attempting fractionated therapy in these larger-volume tumors. To limit dosage to the visual pathways to less than $8 \mathrm{~Gy}$, Skeie et al. treated their series of 100 patients with CSMs (which included some atypical meningiomas) with a mean dose to the periphery of $12.4 \mathrm{~Gy} .{ }^{48}$ They noted a lower rate of tumor control $(84 \%)$ than most other modern series. Specifically, they found that the tumors that received a suboptimal radiation dose to a part of the lesion had the highest rate of recurrence (9 of 23,39\%). Similarly, Hasegawa et al., in their series of radiosurgically treated CSMs, found that the subsets of larger tumors ( $>$ than 36 $\mathrm{cm}^{3}$ and $54 \mathrm{~cm}^{3}$ ) treated with lower tumor margin doses in the range of 10-12 Gy had 5- and 10-year tumor control rates of $87 \%$ and $73 \%$, respectively. ${ }^{14} \mathrm{~A}$ higher posttreatment recurrence rate indicates a higher likelihood of needing additional treatment, either further radiation treatment or postradiation microsurgical treatment, which has been shown to carry a higher rate of postoperative complications for CSMs than in primary surgical cases. Finally, Metellus et al. compared their CSMs treated with fractionated radiotherapy with those treated with GKRS; ${ }^{29}$ the fractionated treatment subgroup tended to have larger lesions, and the degree of radiological treatment response was greater in the radiosurgical cohort.

\section{Conclusions}

Modern neurosurgical treatment of CSMs is predicated on an understanding of the microsurgical anatomy of the cavernous sinus, specifically how meningiomas can abut, partially invade, or completely infiltrate the sinus and its critical structures. The development and implementation of skull base microsurgical approaches to CSMs in the 1980s and 1990s has demonstrated that a surgical cure is untenable for the majority of CSMs and that exploration of the cavernous sinus proper involves a high rate of cranial nerve and vascular morbidity. Subsequently, in the late 1990s and early 2000s, GKRS emerged as an effective and safe therapeutic option for CSMs, both as a primary or as an adjunctive treatment. The goal of modern therapy for CSMs should be maximizing tumor growth control while minimizing tumor-related and treatmentrelated morbidity. This goal requires a multidisciplinary strategy, involving neurosurgery and radiation oncology, which is individualized to the patient's clinical status, tumor histology, and tumor anatomy.

\section{Acknowledgment}

We greatly appreciate the assistance of Suzanne Truex with the Department of Neurological Surgery at the University of Texas Southwestern Medical Center for the development of the illustrations for the paper.

\section{Disclosure}

The authors report no conflict of interest concerning the materials or methods used in this study or the findings specified in this paper. 


\section{Evolution in treatment of cavernous sinus meningiomas}

Author contributions to the study and manuscript preparation include the following. Conception and design: Klinger, Lewis, Barnett. Acquisition of data: Klinger. Drafting the article: Klinger, Flores. Critically revising the article: all authors. Reviewed submitted version of manuscript: Klinger, Barnett. Study supervision: Barnett.

\section{References}

1. Abdel-Aziz KM, Froelich SC, Dagnew E, Jean W, Breneman JC, Zuccarello M, et al: Large sphenoid wing meningiomas involving the cavernous sinus: conservative surgical strategies for better functional outcomes. Neurosurgery 54:1375-1384, 2004

2. Bondy M, Ligon BL: Epidemiology and etiology of intracranial meningiomas: a review. J Neurooncol 29:197-205, 1996

3. Brell M, Villà S, Teixidor P, Lucas A, Ferrán E, Marín S, et al: Fractionated stereotactic radiotherapy in the treatment of exclusive cavernous sinus meningioma: functional outcome, local control, and tolerance. Surg Neurol 65:28-34, 2006

4. Couldwell WT, Kan P, Liu JK, Apfelbaum RI: Decompression of cavernous sinus meningioma for preservation and improvement of cranial nerve function. Technical note. J Neurosurg 105:148-152, 2006

5. De Jesús O, Sekhar LN, Parikh HK, Wright DC, Wagner DP: Long-term follow-up of patients with meningiomas involving the cavernous sinus: recurrence, progression, and quality of life. Neurosurgery 39:915-920, 1996

6. DeMonte F, Smith HK, al-Mefty O: Outcome of aggressive removal of cavernous sinus meningiomas. J Neurosurg 81: 245-251, 1994

7. Dufour H, Muracciole X, Métellus P, Régis J, Chinot O, Grisoli F: Long-term tumor control and functional outcome in patients with cavernous sinus meningiomas treated by radiotherapy with or without previous surgery: is there an alternative to aggressive tumor removal? Neurosurgery 48:285-296, 2001

8. el-Kalliny M, van Loveren H, Keller JT, Tew JM Jr: Tumors of the lateral wall of the cavernous sinus. J Neurosurg 77:508514,1992

9. Gay E, Lages E, Ramus C, Guttin A, El Atifi M, Dupré I, et al: The heterogeneity of meningioma revealed by multiparameter analysis: infiltrative and non-infiltrative clinical phenotypes. Int J Oncol 38:1287-1297, 2011

10. Goel A: The extradural approach to lesions involving the cavernous sinus. Br J Neurosurg 11:134-138, 1997

11. Goel A, Kothari M: Editorial. Cavernous sinus meningiomas. J Neurosurg 113:1085-1086, 2010

12. Goldsmith BJ, Wara WM, Wilson CB, Larson DA: Postoperative irradiation for subtotally resected meningiomas. A retrospective analysis of 140 patients treated from 1967 to 1990 . J Neurosurg 80:195-201, 1994

13. Hakuba A, Tanaka K, Suzuki T, Nishimura S: A combined orbitozygomatic infratemporal epidural and subdural approach for lesions involving the entire cavernous sinus. J Neurosurg 71:699-704, 1989

14. Hasegawa T, Kida Y, Yoshimoto M, Koike J, Iizuka H, Ishii D: Long-term outcomes of Gamma Knife surgery for cavernous sinus meningioma. J Neurosurg 107:745-751, 2007

15. Hashimoto N, Rabo CS, Okita Y, Kinoshita M, Kagawa N, Fujimoto Y, et al: Slower growth of skull base meningiomas compared with non-skull base meningiomas based on volumetric and biological studies. Clinical article. J Neurosurg 116:574-580, 2012

16. Iwai Y, Yamanaka K, Ishiguro T: Gamma knife radiosurgery for the treatment of cavernous sinus meningiomas. Neurosurgery 52:517-524, 2003

17. Iwai Y, Yamanaka K, Nakajima H: Two-staged gamma knife radiosurgery for the treatment of large petroclival and cavernous sinus meningiomas. Surg Neurol 56:308-314, 2001

18. Jääskeläinen J: Seemingly complete removal of histologically benign intracranial meningioma: late recurrence rate and factors predicting recurrence in 657 patients. A multivariate analysis. Surg Neurol 26:461-469, 1986

19. Jänisch W, Staneczek W: [Epidemiology of tumors of the central nervous system-influence of the autopsy rate on the incidence rate.] Arch Geschwulstforsch 58:51-55, 1988 (Ger)

20. Kawase T, Toya S, Shiobara R, Mine T: Transpetrosal approach for aneurysms of the lower basilar artery. J Neurosurg 63:857-861, 1985

21. Kim DK, Grieve J, Archer DJ, Uttley D: Meningiomas in the region of the cavernous sinus: a review of 21 patients. Br $\mathbf{J}$ Neurosurg 10:439-444, 1996

22. Klink DF, Sampath P, Miller NR, Brem H, Long DM: Longterm visual outcome after nonradical microsurgery patients with parasellar and cavernous sinus meningiomas. Neurosurgery 47:24-32, 2000

23. Kobayashi T, Kida Y, Mori Y: Long-term results of stereotactic gamma radiosurgery of meningiomas. Surg Neurol 55: 325-331, 2001

24. Kondziolka D, Mathieu D, Lunsford LD, Martin JJ, Madhok R, Niranjan A, et al: Radiosurgery as definitive management of intracranial meningiomas. Neurosurgery 62:53-60, 2008

25. Kotapka MJ, Kalia KK, Martinez AJ, Sekhar LN: Infiltration of the carotid artery by cavernous sinus meningioma. J Neurosurg 81:252-255, 1994

26. Larson JJ, van Loveren HR, Balko MG, Tew JM Jr: Evidence of meningioma infiltration into cranial nerves: clinical implications for cavernous sinus meningiomas. J Neurosurg 83: 596-599, 1995

27. Maruyama K, Shin M, Kurita H, Kawahara N, Morita A, Kirino T: Proposed treatment strategy for cavernous sinus meningiomas: a prospective study. Neurosurgery 55:1068-1075, 2004

28. Mathiesen T, Lindquist C, Kihlström L, Karlsson B: Recurrence of cranial base meningiomas. Neurosurgery 39:2-9, 1996

29. Metellus P, Regis J, Muracciole X, Fuentes S, Dufour H, Nanni I, et al: Evaluation of fractionated radiotherapy and gamma knife radiosurgery in cavernous sinus meningiomas: treatment strategy. Neurosurgery 57:873-886, 2005

30. Morita A, Coffey RJ, Foote RL, Schiff D, Gorman D: Risk of injury to cranial nerves after gamma knife radiosurgery for skull base meningiomas: experience in 88 patients. J Neurosurg 90:42-49, 1999

31. Nakamura M, Roser F, Michel J, Jacobs C, Samii M: The natural history of incidental meningiomas. Neurosurgery 53: 62-71, 2003

32. Nicolato A, Foroni R, Alessandrini F, Maluta S, Bricolo A, Gerosa M: The role of Gamma Knife radiosurgery in the management of cavernous sinus meningiomas. Int J Radiat Oncol Biol Phys 53:992-1000, 2002

33. O'Sullivan MG, van Loveren HR, Tew JM Jr: The surgical resectability of meningiomas of the cavernous sinus. Neurosurgery 40:238-247, 1997

34. Olivero WC, Lister JR, Elwood PW: The natural history and growth rate of asymptomatic meningiomas: a review of $60 \mathrm{pa}-$ tients. J Neurosurg 83:222-224, 1995

35. Oya S, Kim SH, Sade B, Lee JH: The natural history of intracranial meningiomas. Clinical article. J Neurosurg 114: 1250-1256, 2011

36. Pamir MN, Kiliç T, Bayrakli F, Peker S: Changing treatment strategy of cavernous sinus meningiomas: experience of a single institution. Surg Neurol 64 (Suppl 2):S58-S66, 2005

37. Parkinson D: Lateral sellar compartment: history and anatomy. J Craniofac Surg 6:55-68, 1995

38. Parkinson D: A surgical approach to the cavernous portion of the carotid artery. Anatomical studies and case report. J Neurosurg 23:474-483, 1965

39. Parkinson D, Ramsay RM: Carotid cavernous fistula with pulsating exophthalmos: a fortuitous cure. Can J Surg 6:191195, 1963 


\section{R. Klinger et al.}

40. Pichierri A, Santoro A, Raco A, Paolini S, Cantore G, Delfini R: Cavernous sinus meningiomas: retrospective analysis and proposal of a treatment algorithm. Neurosurgery 64:10901101, 2009

41. Pollock BE, Stafford SL, Link MJ, Garces YI, Foote RL: Single-fraction radiosurgery of benign cavernous sinus meningiomas. Clinical article. J Neurosurg 119:675-682, 2013

42. Radhakrishnan K, Mokri B, Parisi JE, O'Fallon WM, Sunku $\mathrm{J}$, Kurland LT: The trends in incidence of primary brain tumors in the population of Rochester, Minnesota. Ann Neurol 37:67-73, 1995

43. Shaffrey ME, Dolenc VV, Lanzino G, Wolcott WP, Shaffrey CI: Invasion of the internal carotid artery by cavernous sinus meningiomas. Surg Neurol 52:167-171, 1999

44. Shin M, Kurita H, Sasaki T, Kawamoto S, Tago M, Kawahara N, et al: Analysis of treatment outcome after stereotactic radiosurgery for cavernous sinus meningiomas. J Neurosurg 95:435-439, 2001

45. Shrieve DC, Hazard L, Boucher K, Jensen RL: Dose fractionation in stereotactic radiotherapy for parasellar meningiomas: radiobiological considerations of efficacy and optic nerve tolerance. J Neurosurg 101 (Suppl 3):390-395, 2004

46. Shrivastava RK, Sen C, Costantino PD, Della Rocca R: Sphenoorbital meningiomas: surgical limitations and lessons learned in their long-term management. J Neurosurg 103:491-497, 2005

47. Sindou M, Wydh E, Jouanneau E, Nebbal M, Lieutaud T: Longterm follow-up of meningiomas of the cavernous sinus after surgical treatment alone. J Neurosurg 107:937-944, 2007

48. Skeie BS, Enger PO, Skeie GO, Thorsen F, Pedersen PH: Gamma knife surgery of meningiomas involving the cavernous sinus: long-term follow-up of 100 patients. Neurosurgery 66:661-669, 2010

49. Sughrue ME, Kane AJ, Shangari G, Rutkowski MJ, McDer- mott MW, Berger MS, et al: The relevance of Simpson Grade I and II resection in modern neurosurgical treatment of World Health Organization Grade I meningiomas. Clinical article. J Neurosurg 113:1029-1035, 2010

50. Sughrue ME, Rutkowski MJ, Aranda D, Barani IJ, McDermott MW, Parsa AT: Factors affecting outcome following treatment of patients with cavernous sinus meningiomas. Clinical article. J Neurosurg 113:1087-1092, 2010.

51. Suzuki M, Mizoi K, Yoshimoto T: Should meningiomas involving the cavernous sinus be totally resected? Surg Neurol 44:3-13, 1995

52. Tishler RB, Loeffler JS, Lunsford LD, Duma C, Alexander E III, Kooy HM, et al: Tolerance of cranial nerves of the cavernous sinus to radiosurgery. Int J Radiat Oncol Biol Phys 27:215-221, 1993

53. Walker AE, Robins M, Weinfeld FD: Epidemiology of brain tumors: the national survey of intracranial neoplasms. Neurology 35:219-226, 1985

54. Yasuda A, Campero A, Martins C, Rhoton AL Jr, de Oliveira E, Ribas GC: Microsurgical anatomy and approaches to the cavernous sinus. Neurosurgery 56 (1 Suppl):4-27, 2005

55. Zada G, Pagnini PG, Yu C, Erickson KT, Hirschbein J, Zelman V, et al: Long-term outcomes and patterns of tumor progression after gamma knife radiosurgery for benign meningiomas. Neurosurgery 67:322-329, 2010

Manuscript submitted August 15, 2013.

Accepted September 17, 2013.

Please include this information when citing this paper: DOI: 10.3171/2013.9.FOCUS13345.

Address correspondence to: Daniel R. Klinger, M.D., Department of Neurological Surgery, 5323 Harry Hines Blvd., Dallas, TX 75390-8855. email: danielrklinger@gmail.com. 